\title{
Beclin-1 knockdown decreases proliferation, invasion and migration of Ewing sarcoma SK-ES-1 cells via inhibition of MMP-9
}

\author{
CONGLIN YE*, XIAOLONG YU*, XUQIANG LIU, PING ZHAN, TAO NIE, \\ RUNSHENG GUO, HUCHENG LIU, MIN DAI and BIN ZHANG
}

\begin{abstract}
Department of Orthopedics, The First Affiliated Hospital of Nanchang University, Artificial Joints Engineering and Technology Research Center of Jiangxi, Nanchang, Jiangxi 330006, P.R. China
\end{abstract}

Received January 24, 2017; Accepted November 2, 2017

DOI: $10.3892 / \mathrm{ol} .2017 .7667$

\begin{abstract}
Although Beclin-1, a well-known key regulator of autophagy, has been demonstrated to serve a function in a number of disorders, including cancer, aging and degenerative diseases, its biological function in Ewing sarcoma (ES) remains unresolved. The objective of the present study was to determine the in vitro effect of Beclin-1 knockdown on the growth and malignant phenotype of ES SK-ES-1 cells, which have increased endogenous expression of Beclin-1 compared with RD-ES cells, and to investigate the underlying molecular mechanism. Cell proliferation, invasion and migration were investigated using CCK-8, Boyden chamber Transwell, and wound healing assays, respectively. Western blot analysis was used to detect expression levels of matrix metalloproteinase (MMP)-2 and MMP-9, which are associated with the malignant phenotype. Beclin-1 knockdown significantly inhibited proliferation, invasion and migration of SK-ES-1 cells. Western blot analysis revealed that Beclin-1 knockdown caused a significant reduction in the expression of MMP-9; no marked changes in MMP-2 expression were observed in the si-Beclin-1 group compared with the control group. The results of the present study suggest that Beclin-1 serves a function in proliferation, tumor progression and inhibition of autophagy in ES, and demonstrates it's potential as a target to increase the efficacy of anticancer agents.
\end{abstract}

Correspondence to: Professor Bin Zhang or Professor Min Dai, Department of Orthopedics, The First Affiliated Hospital of Nanchang University, Artificial Joints Engineering and Technology Research Center of Jiangxi, 17 Yong Wai Zheng Street, Nanchang, Jiangxi 330006, P.R. China

E-mail: 1294522934@qq.com

E-mail: daimin@medmail.com.cn

*Contributed equally

Key words: autophagy, Beclin-1, Ewing sarcoma, matric metalloproteinase-9, invasion, migration

\section{Introduction}

Ewing sarcoma (ES) is the second most common sarcoma of bone in children and young adults (1). It is an aggressive and highly metastatic tumor. In total, $\sim 1 / 3$ of patients with ES present with metastasis at diagnosis, with lung tissue and bone marrow being the most common sites of metastasis, resulting in poor prognosis (2). Treatment and prognosis of patients with ES are determined by the presence of metastases, among other factors. The 5-year survival rate of patients with metastases ranges between 20 and $45 \%$, depending on location, compared with between 60 and 70\% in those with localized disease (2). Thus, novel therapeutic targets, innovative approaches to therapy and improved understanding of the metastatic mechanism are necessary to improve the outcome for patients with metastatic ES.

Autophagy is a highly conserved process that contributes to maintaining cellular homeostasis via quality control of proteins and organelles. Under conditions of metabolic stress, autophagy provides nutrients and energy essential for cell survival (3-5). Beclin-1 is a B-cell lymphoma 2 (Bcl-2) homology 3 domain-only protein that is required for the formation of autophagosomes, which are utilized in the initiation of autophagy (6-8). It has been reported that cell autophagy is associated with tumor initiation and progression, and serves a function in cell signal regulation in tumors (9-11). To date, the exact effects of autophagy on the biological behavior of ES cells have not been fully resolved.

In the present study, SK-ES-1 cells were transfected with small interfering (si)RNA against Beclin-1 in order to investigate the effects of Beclin-1 knockdown on cell proliferation, invasion and migration, and to determine the underlying molecular mechanisms. To the best of our knowledge, this is the first study to report the effects of Beclin-1 knockdown on the behavior of ES cells.

\section{Materials and methods}

Materials and reagents. The SK-ES-1 and RD-ES human ES cell lines were obtained from the American Type Culture Collection (ATCC; Manassas, VA, USA). RPMI-1640 medium, 
fetal bovine serum (FBS), PBS, dimethyl sulfoxide and Cell Counting Kit-8 (CCK-8) were provided by Beijing Transgen Biotech Co., Ltd. (Beijing, China). Antibodies against Beclin-1 (ab207612, 1:1,000), matrix metalloproteinase (MMP)-2 (ab92536, 1:1,000), MMP-9 (ab194314, 1:1,000) and $\beta$-actin (ab8227, 1:1,000) were all purchased from Abcam (Cambridge, $\mathrm{UK})$. Goat anti-rabbit IgG $(\mathrm{H}+\mathrm{L})$, horseradish peroxidase-conjugated secondary antibodies (HS101-01, 1:2,000) were both purchased from Beijing Transgen Biotech Co., Ltd. Lipofectamine $^{\circledR} 2000$ and OPTI-MEM were both purchased from Invitrogen (Thermo Fisher Scientific, Inc., Waltham, MA, USA). Matrigel was purchased from BD Biosciences (San Jose, CA, USA). The Transwell invasion chambers were purchased from Costar (Cambridge, MA, USA). Crystal violet staining solution was purchased from Beyotime Institute of Biotechnology (Haimen, China). si-Beclin-1 against the BECLIN-1 gene (NM_003766) and control siRNA (si-CON) were constructed by Shanghai GeneChem Co., Ltd. (Shanghai, China).

Cell culture and transfection. SK-ES-1 and RD-ES cells were cultured in RPMI-1640 medium supplemented with $10 \%$ (v/v) FBS, $100 \mathrm{U} / \mathrm{ml}$ penicillin and $100 \mu \mathrm{g} / \mathrm{ml}$ streptomycin. Cells were incubated in a humidified atmosphere containing $5 \% \mathrm{CO}_{2}$ at $37^{\circ} \mathrm{C}$. All cells used in the present study were subjected to $<20$ cell passages. SK-ES-1 cells at logarithmic phase were seeded at a density of $3 \times 10^{5}$ cells/well in a 6 -well plate for $24 \mathrm{~h}$ prior to transfection. Lipofectamine $2000(10 \mu \mathrm{l}$ diluted in $250 \mu \mathrm{l}$ OPTI-MEM) was used for the transfection of $4 \mu \mathrm{g}$ si-Beclin-1 or empty vector diluted in $250 \mu$ l OPTI-MEM, followed by incubation of the samples for $20 \mathrm{~min}$ at room temperature. The plasmid DNA-Lipofectamine 2000 complex was then added into $500 \mu \mathrm{l}$ OPTI-MEM and incubated at $37^{\circ} \mathrm{C}$ with $5 \% \mathrm{CO}_{2}$ in an incubator for $6 \mathrm{~h}$. Subsequently, the medium was replaced and the cells were incubated for 24 or $48 \mathrm{~h}$ in RPMI-1640 medium supplemented with $10 \%$ FBS prior to use in the corresponding experiments, which included a blank control group (non-transfected SK-ES-1 cells), a negative control group (SK-ES-1 cells transfected with blank plasmid, i.e., si-CON) and an experimental group (SK-ES-1 cells transfected with si-Beclin-1). Transfected cells were collected at 24 or $48 \mathrm{~h}$ post-transfection and used in subsequent experiments.

Cell proliferation assay. Cell growth was determined using the CCK-8 assay. In brief, cells infected with si-Beclin-1 or si-CON and non-transfected SK-ES-1 cells were incubated in 96-well plates at a density of $3 \times 10^{3}$ cells/well. Cells were treated with $10 \mu \mathrm{l} \mathrm{CCK}-8$ reagent at 24,48 and $72 \mathrm{~h}$ and then measured at $450 \mathrm{~nm}$ using a Universal Microplate reader (EL800; Bio-Tek Instruments Inc., Winooski, VT, USA).

Boyden chamber Transwell assays. The invasive capacity of SK-ES-1 cells was detected via Matrigel-coated Transwell cell culture chambers ( $8 \mu \mathrm{m}$ pore size). Following transfection for $24 \mathrm{~h}$, SK-ES-1 cells of the three different groups were collected and suspended in serum-free medium. Isolated cells were then added to the upper chamber of the Transwell insert at a density of $4 \times 10^{4}$ cells/well and the lower wells were filled with complete growth medium supplemented with $10 \%$ FBS. All samples were incubated for $24 \mathrm{~h}$ in a $\mathrm{CO}_{2}$ incubator.
Non-invading cells (on the upper membrane surface) were removed using a cotton swab and invading cells (on the lower membrane surface) were fixed with $95 \%$ ethanol for $15 \mathrm{~min}$ at $25^{\circ} \mathrm{C}$, stained with $0.1 \%$ crystal violet staining solution for $20 \mathrm{~min}$ at $25^{\circ} \mathrm{C}$, then counted under a phase-contrast microscope in three random fields (magnification, $\mathrm{x} 200$ ).

Wound healing assays. Migration of SK-ES-1 cells was measured using wound healing assays. After $24 \mathrm{~h}$ of transfection, SK-ES-1 cells of the three different groups were seeded at a density of $5 \times 10^{5}$ cells/well in a 6 -well culture plate to form a confluent monolayer. Cells were wounded with a sterile $100 \mu \mathrm{l}$ pipette tip. All cells in the plates were incubated in fresh RPMI-1640 medium with 10\% FBS for $24 \mathrm{~h}$. Then scratch wounds were observed using a phase-contrast microscope and images were captured of each wound.

Western blot analysis. Following transfection, SK-ES-1 cells of the three different groups were seeded in 6-well plates at a concentration of $3 \times 10^{5}$ cells/well and incubated in RPMI-1640 medium with $10 \%$ FBS for $48 \mathrm{~h}$. The cells were collected and lysed in radioimmunoprecipitation assay buffer containing phenylmethane sulfonyl fluoride and phosphatase inhibitor cocktail (Sigma Aldrich; Merck KGaA, Darmstadt, Germany). Each sample was centrifuged at $17,105.6 \mathrm{xg}$ for $10 \mathrm{~min}$ at $4^{\circ} \mathrm{C}$ using a Universal 320R centrifuge (Andreas Hettich $\mathrm{GmbH}$ \& Co. KG, Tuttlingen, Germany), to remove cell debris and collect the supernatant for immunoblotting. Protein concentrations were calculated using a bicinchoninic acid assay kit (Beijing Transgen Biotech Co., Ltd.) according to the manufacturer's instructions with bovine serum albumin as the relative standard. Proteins $(10 \mu \mathrm{l})$ were loaded and separated using SDS-PAGE (10\% gel, $100 \mathrm{~V}$ for $2 \mathrm{~h}$ under reducing conditions). Following electrophoresis, the proteins were transferred to polyvinylidene fluoride (PVDF) membranes in a tris-glycine transfer buffer and incubated with antibodies against $\beta$-actin, Beclin-1, MMP-2 and MMP-9 overnight at $4^{\circ} \mathrm{C}$. The PVDF membranes were washed in Tris-buffered saline Tween-20 (TBST) three times. Secondary HRP-conjugated antibodies were added at 1:2,000 dilution and incubated for $2 \mathrm{~h}$ at $25^{\circ} \mathrm{C}$. The PVDF membranes were washed a further three times in TBST. Immunoreactive proteins were detected using an enhanced chemiluminescence system (GE Healthcare, Chicago, IL, USA) according to the manufacturer's instructions followed by exposure to X-ray films. Western blotting data was quantified using ImageJ software (version 7.0; National Institutes of Health, Bethesda, MD, USA).

Statistical analysis. Data were analyzed using the SPSS package for Windows (version 19.0; IBM Corp., Armonk, NY, USA). Quantitative data are expressed as mean \pm standard deviation. Statistical analysis was performed using a one-way analysis of variance with the Student-Newman-Keuls method as a post hoc test. $\mathrm{P}<0.05$ was considered to indicate a statistically significant difference.

\section{Results}

Endogenous expression of Beclin-1 in the human ES cell lines. Endogenous expression of Beclin-1 in the human ES SK-ES-1 
A

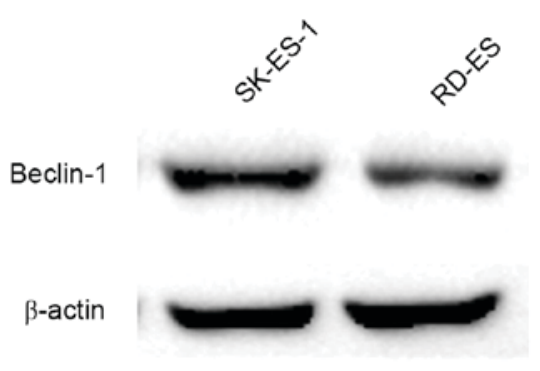

C

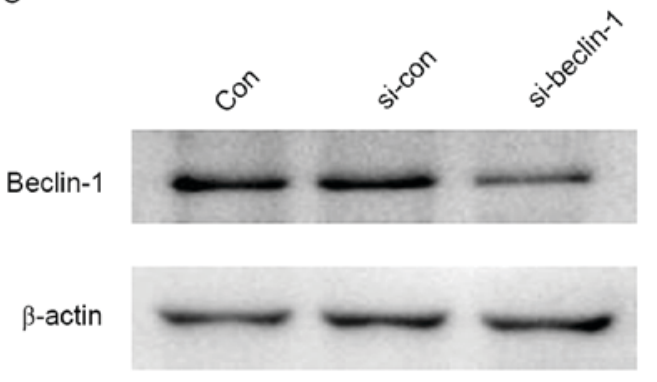

B

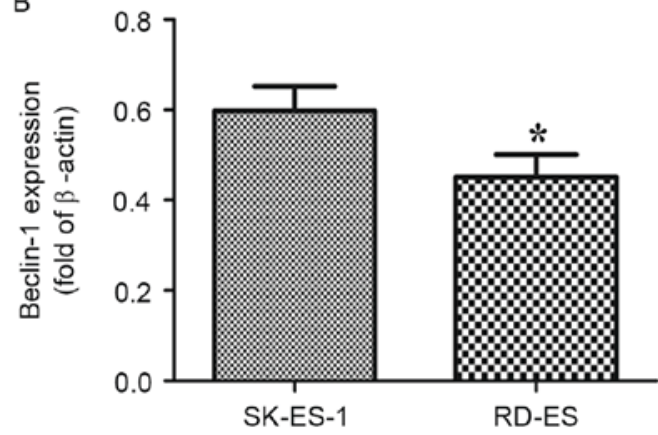

D

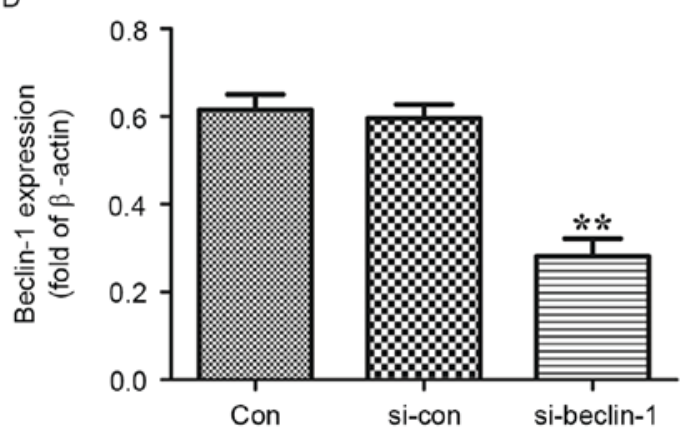

Figure 1. Endogenous expression of Beclin-1 in the SK-ES-1 and RD-ES cell lines was evaluated using western blot analysis. (A) Expression of Beclin-1 was markedly increased in the SK-ES-1 cell line compared with the RD-ES cell line. (B) Quantification of the western blotting confirmed that the expression of Beclin-1 was significantly decreased in RD-ES cells compared with SK-ES-1 cells. (C) Protein levels of Beclin-1 were determined using western blotting once SK-ES-1 cells were transfected with si-beclin-1 or si-con vectors for $48 \mathrm{~h}$. (D) Beclin-1 expression was significantly decreased in the si-beclin-1 group compared with the blank control group. ${ }^{*} \mathrm{P}<0.05,{ }^{* *} \mathrm{P}<0.01$ compared with control group. Con, blank control group; si-con, SK-ES-1 cells transfected with blank plasmid; si-beclin-1, SK-ES-1 cells with Beclin-1 knocked down.

and RD-ES cell lines was evaluated using western blot analysis. As presented in Fig. 1A and B, expression of Beclin-1 was significantly increased in the SK-ES-1 cell line compared with the RD-ES cell line $(\mathrm{P}<0.05)$. Thus, the SK-ES-1 cell line was used for Beclin-1 knockdown.

si-Beclin-1 significantly decreases the expression of Beclin-1 in SK-ES-1 cells. Protein levels of Beclin-1 were determined using western blotting following transfection of SK-ES-1 cells with si-Beclin-1 or si-CON vectors for $48 \mathrm{~h}$. As presented in Fig. 1C and D, Beclin-1 expression was significantly decreased in the si-Beclin-1 group compared with the blank control group $(\mathrm{P}<0.01)$

Beclin-1 knockdown inhibits the proliferation of SK-ES-1 cells. The effect of Beclin-1 knockdown on SK-ES-1 cell growth was determined using a CCK-8 assay. As presented in Fig. 2A, knockdown of Beclin-1 significantly suppressed the growth of SK-ES-1 cells ( $\mathrm{P}<0.05$ at $24 \mathrm{~h}$ and $\mathrm{P}<0.01$ at $48 \mathrm{~h}$ ).

Beclin-1 knockdown represses the invasion and migration of SK-ES-1 cells. Transwell and wound healing assays were conducted to confirm the effect of Beclin-1 knockdown on the invasion and migration of SK-ES-1 cells. Representative micrographs of Transwell filters are presented in Fig. 2B. The invasive cell count, also presented in Fig. 2B, demonstrated that the invasive potential was significantly decreased in the si-Beclin-1 group relative to the blank control group $(\mathrm{P}<0.01)$. Furthermore, Beclin-1 knockdown resulted in a decrease in migration capability, as presented in Fig. 2C and D $(\mathrm{P}<0.01)$.

A western blot assay was performed to investigate the effect of Beclin-1 knockdown on the expression of MMP-2 and MMP-9, since it is generally acknowledged that they serve functions in tumor invasion, and metastasis (12-14). As presented in Fig. 3A and B, MMP-9 expression was significantly decreased in the si-Beclin-1 group compared with the Con group $(\mathrm{P}<0.01)$; however, no significant difference in the expression of MMP-2 was observed between the two groups. These results suggest that Beclin-1 knockdown may inhibit invasion and metastasis of SK-ES-1 cells via downregulation of the expression of MMP-9.

\section{Discussion}

ES is an aggressive bone and soft tissue malignant tumor that primarily affects children, and young adults (15). In previous years, the overall survival rate has risen markedly for patients who present with localized disease, owing to development of multi-agent systemic chemotherapy and aggressive local control methods, which have resulted in five-year event-free survival rates of $70-80 \%$ in these patients $(15,16)$. However, for the $25 \%$ of patients who present with metastatic disease, the prognosis is poor and event-free survival rate for these patients remains $<25 \%$ (15). Thus, novel therapeutic targets and increased understanding of the metastatic mechanism of ES are required to achieve an improved outcome for these patients. 
A

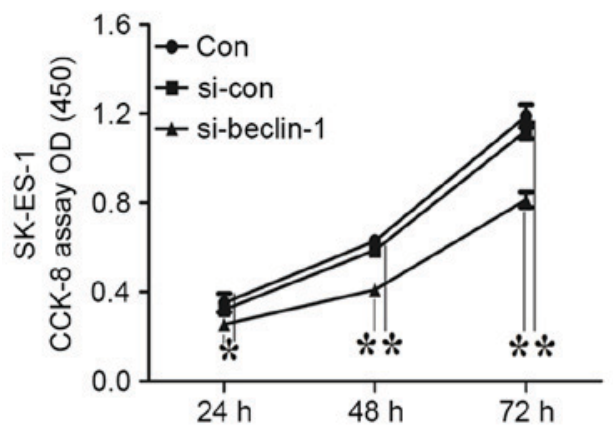

C
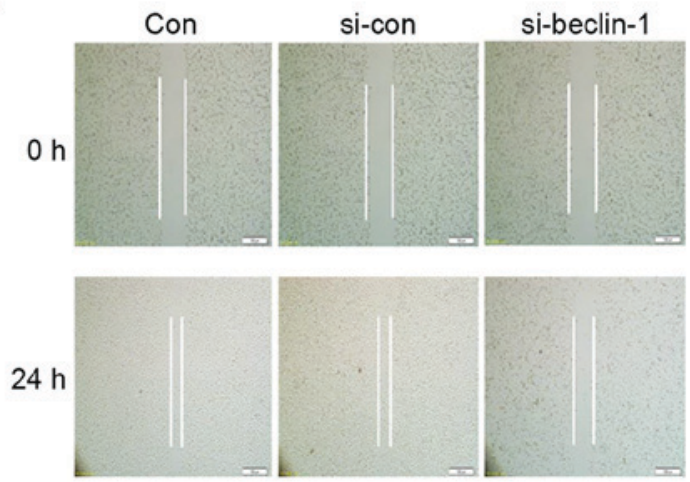

B
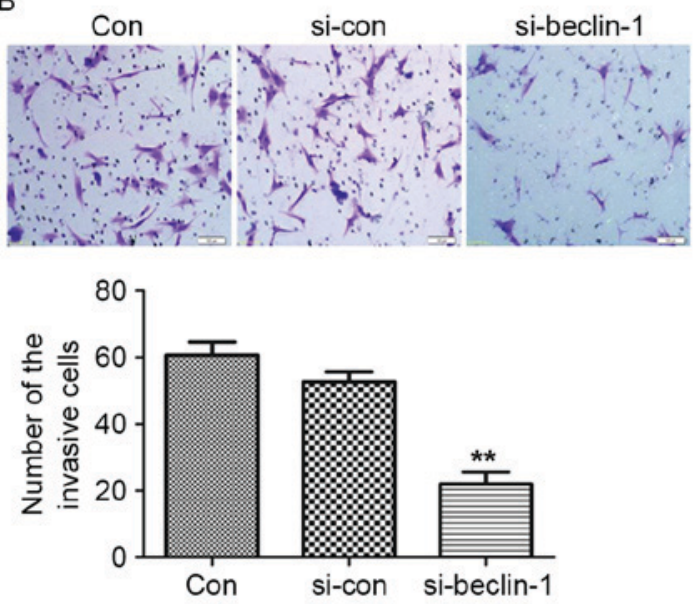

D

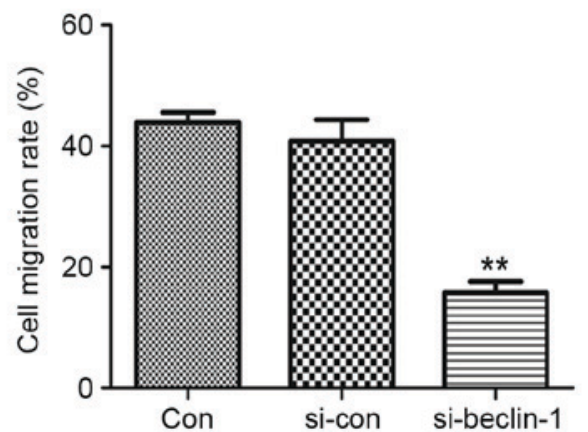

Figure 2. Knockdown of Beclin-1 suppressed proliferation, invasion and migration of SK-ES-1 cells. (A) CCK-8 assay was performed to examine SK-ES-1 cell proliferation. Knockdown of Beclin-1 significantly inhibited SK-ES-1 cell proliferation. (B) A Matrigel-coated Transwell assay was conducted to confirm the invasion of SK-ES-1 cells (magnification, x100). Knockdown of Beclin-1 significantly repressed the invasion of SK-ES-1 cells. (C) A wound healing assay was conducted to determine the migration of SK-ES-1 cells (magnification, x40). (D) Knockdown of Beclin-1 significantly repressed the migration of SK-ES-1 cells. ${ }^{* *} \mathrm{P}<0.01$ compared with the control group. CCK-8, Cell Counting Kit-8; con, blank control group; si-con, SK-ES-1 cells transfected with blank plasmid; si-beclin-1, SK-ES-1 cells with beclin-1 knocked down.

A

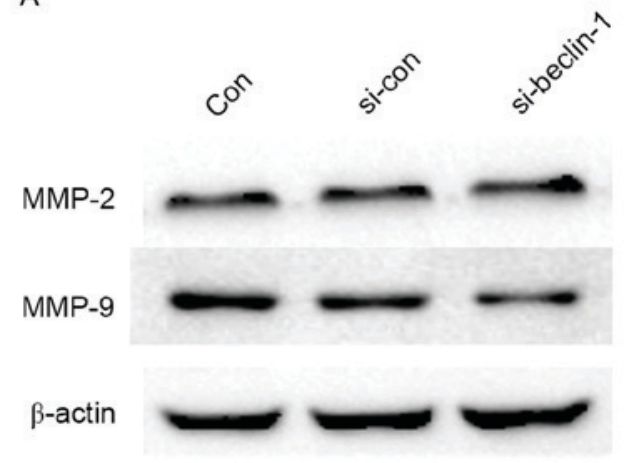

B

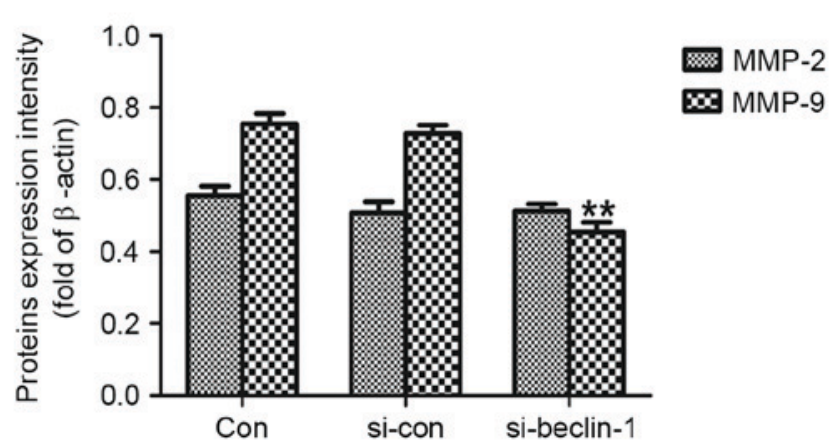

Figure 3. Beclin-1 knockdown decreases MMP-9 expression. (A) Western blot analysis was performed to investigate the effect of Beclin-1 knockdown on the expression of MMP-2 and MMP-9, since it is generally acknowledged that they are associated with tumor invasion and metastasis. (B) MMP-9 expression was significantly decreased in the si-beclin-1 group compared with the Con group, although no significant difference in the expression of MMP-2 was observed. ${ }^{* *} \mathrm{P}<0.01$ compared with the control group. MMP, matrix metalloproteinase; con, blank control group; si-con, SK-ES-1 cells transfected with blank plasmid; si-beclin-1, SK-ES-1 cells with beclin-1 knocked down.

The function of autophagy in cancer has been highlighted in previous years. Autophagy serves a function in cell cycle regulation, apoptosis, angiogenesis and other aspects of tumor initiation and progression (17). Increasing evidence suggests that autophagy contributes to the malignant phenotype in a number of tumors, including lung adenocarcinoma, ovarian carcinoma and esophageal squamous cell carcinoma (18-20). Autophagy-related protein six, known as Beclin-1, the first confirmed mammalian autophagic gene, has been demonstrated to initiate autophagosome formation through binding 
to a class III phosphatidylinositol-3-OH kinase (Vps34). Beclin-1 upregulates autophagy by combining with Vps34, and other positive and negative co-factors, including Bcl-2/B-cell lymphoma extra-large, Vps15, Beclin-1-associated autophagy-related key regulator, Autophagy and Beclin-1 regulator 1 , High mobility group box 1 , and Survivin to form the Beclin-1 interactome (21). Beclin-1 dysfunction has been identified in a number of disorders, including cancer, aging and degenerative diseases; for example, overexpression of Beclin-1 markedly promotes autophagic cell death in leukemia cells (22). Additionally, Beclin-1 knockdown using siRNA protects cells from sorafenib-induced autophagic cell death in hepatocellular carcinoma cells (23). On the contrary, autophagy promotes tumor survival and progression in pancreatic cancer (24). However, the exact function of Beclin-1 in the proliferation and malignant phenotype of ES cells remains unclear.

In the present study, it was demonstrated that Beclin-1 knockdown inhibited proliferation, invasion and migration in SK-ES-1 cells. The simultaneous decline in the expression of MMP-9, which is generally established to be closely associated with tumor invasion and metastasis, suggested that Beclin-1 knockdown may inhibit invasion and migration of SK-ES-1 cells through downregulating the expression of MMP-9.

Taken together, the results of the present study suggest that Beclin-1 knockdown may suppress the growth and malignant phenotype of SK-ES-1 cells by inhibition of MMP-9. Thus Beclin-1 is a promising therapeutic target for treatment of ES, particularly in patients that present with metastasis. Further experiments on the in vitro effects of Beclin-1 knockdown on apoptosis of ES cells may further resolve its viability as a therapeutic target.

\section{Acknowledgements}

The present study was supported by The Foundation of Health Department of Jiangxi Province (grant no. 2016A073) and Gan-Po Talents Project 555 of Jiangxi Province.

\section{References}

1. Jemal A, Bray F, Center MM, Ferlay J, Ward E and Forman D: Global cancer statistics. CA Cancer J Clin 61: 69-90, 2011.

2. Gaspar N, Hawkins DS, Dirksen U, Lewis IJ, Ferrari S, Le Deley MC, Kovar H, Grimer R, Whelan J, Claude L, et al: Ewing sarcoma: Current management and future approaches through collaboration. J Clin Oncol 33: 3036-3046, 2015.

3. Levine B and Klionsky DJ: Development by self-digestion: Molecular mechanisms and biological functions of autophagy. Dev Cell 6: 463-477, 2004.

4. Mizushima N: Autophagy: Process and function. Genes Dev 21: 2861-2873, 2007

5. Mathew R, Karantza-Wadsworth V and White E: Role of autophagy in cancer. Nat Rev 7: 961-967, 2007.
6. Liang XH, Kleeman LK, Jiang HH, Gordon G, Goldman JE, Berry G, Herman B and Levine B: Protection against fatal Sindbis virus encephalitis by beclin, a novel Bcl-2-interacting protein. J Virol 72: 8586-8596, 1998

7. Oberstein A, Jeffrey PD and Shi Y: Crystal structure of the Bcl-XL-Beclin 1 peptide complex: Beclin 1 is a novel BH3-only protein. J Biol Chem 282: 13123-13132, 2007.

8. Liu B, Bao JK, Yang JM and Cheng Y: Targeting autophagic pathways for cancer drug discovery. Chin J Cancer 32: 113-120, 2013.

9. Meijer AJ and Codogno P: Regulation and role of autophagy in mammalian cells. Int J Biochem Cell Biol 36: 2445-2462, 2004.

10. Sun Y, Liu JH, Jin L, Lin SM, Yang Y, Sui YX and Shi H: Over-expression of the Beclin1 gene upregulates chemosensitivity to anti-cancer drugs by enhancing therapy-induced apoptosis in cervix squamous carcinoma CaSki cells. Cancer Lett 294: 204-210, 2010.

11. Jin S and White E: Role of autophagy in cancer: Management of metabolic stress. Autophagy 3: 28-31, 2007.

12. Li H, Zhang K, Liu LH, Ouyang Y, Bu J, Guo HB and Xiao T: A systematic review of matrix metalloproteinase 9 as a biomarker of survival in patients with osteosarcoma. Tumour Biol 35: 5487-5491, 2014.

13. Wang J, Shi Q, Yuan TX, Song QL, Zhang Y, Wei Q, Zhou L, Luo J, Zuo G, Tang M, et al: Matrix metalloproteinase 9 (MMP-9) in osteosarcoma: Review and meta-analysis. Clin Chim Acta 433: 225-231, 2014.

14. Shang HS, Chang JB, Lin JH, Lin JP, Hsu SC, Liu CM, Liu JY, Wu PP, Lu HF, Au MK and Chung JG: Deguelin inhibits the migration and invasion of U-2 OS human osteosarcoma cells via the inhibition of matrix metalloproteinase-2/-9 in vitro. Molecules 19: 16588-16608, 2014.

15. Balamuth NJ and Womer RB: Ewing's sarcoma. Lancet Oncol 11: 184-192, 2010.

16. Gorlick R, Janeway K, Lessnick S, Randall RL and Marina N; COG Bone Tumor Committee: Children's Oncology Group's 2013 blueprint for research: Bone tumors. Pediatr Blood Cancer 60: 1009-1015, 2013.

17. Filippi-Chiela EC, Villodre ES, Zamin LL and Lenz G: Autophagy interplay with apoptosis and cell cycle regulation in the growth inhibiting effect of resveratrol in glioma cells. PLoS One 6: e20849, 2011.

18. Pan B, Chen D, Huang J, Wang R, Feng B, Song H and Chen L: HMGB1-mediated autophagy promotes docetaxel resistance in human lung adenocarcinoma. Mol Cancer 13: 165, 2014.

19. Bao LJ, Jaramillo MC, Zhang ZB, Zheng YX, Yao M, Zhang DD and Yi XF: Nrf2 induces cisplatin resistance through activation of autophagy in ovarian carcinoma. Int J Clin Exp Pathol 7: 1502-1513, 2014.

20. Chen Y, Li X, Wu X, He C, Guo L, Zhang S, Xiao Y, Guo W and Tan B: Autophagy-related proteins LC 3 and Beclin-1 impact the efficacy of chemoradiation on esophageal squamous cell carcinoma. Pathol Res Pract 209: 562-567, 2013.

21. Kang R, Zeh HJ, Lotze MT and Tang D: The Beclin 1 network regulates autophagy and apoptosis. Cell Death Differ 18: 571-580, 2011.

22. Tong Y, You L, Liu H, Li L, Meng H, Qian Q and Qian W: Potent antitumor activity of oncolytic adenovirus expressing beclin-1 via induction of autophagic cell death in leukemia. Oncotarget 4: 860-874, 2013.

23. Tai WT, Shiau CW, Chen HL, Liu CY, Lin CS, Cheng AL, Chen PJ and Chen KF: Mcl-1-dependent activation of Beclin 1 mediates autophagic cell death induced by sorafenib and SC-59 in hepatocellular carcinoma cells. Cell Death Dis 4: e485, 2013.

24. Ko YH, Cho YS, Won HS, Jeon EK, An HJ, Hong SU, Park JH and Lee MA: Prognostic significance of autophagy-related protein expression in resected pancreatic ductal adenocarcinoma. Pancreas 42: 829-835, 2013. 